\title{
Mortality Risk after Dementia Diagnosis by Dementia Type and Underlying Factors: A Cohort of 15,209 Patients based on the Swedish Dementia Registry
}

\author{
Sara Garcia-Ptacek ${ }^{\mathrm{a}, \mathrm{b}, 1, *}$, Bahman Farahmand ${ }^{\mathrm{a}, 1}$, Ingemar Kåreholt ${ }^{\mathrm{c}, \mathrm{d}}$, Dorota Religa ${ }^{\mathrm{e}, \mathrm{f}}$, \\ Maria Luz Cuadrado ${ }^{\mathrm{b}, \mathrm{g}}$ and Maria Eriksdotter ${ }^{\mathrm{a}, \mathrm{e}}$ \\ a Division of Clinical Geriatrics, Department of Neurobiology, Care Sciences and Society, Karolinska Institutet, \\ Stockholm, Sweden \\ ${ }^{\mathrm{b}}$ Department of Medicine, Universidad Complutense, Madrid, Spain \\ ${ }^{\mathrm{c}}$ Aging Research Center, Karolinska Institutet and Stockholm University, Stockholm, Sweden \\ ${ }^{\mathrm{d}}$ Institute of Gerontology, School of Health Sciences, Jönköping University, Jönköping, Sweden \\ ${ }^{\mathrm{e}}$ Department of Geriatric Medicine, Karolinska University Hospital, Karolinska Institutet, Stockholm, Sweden \\ ${ }^{\mathrm{f}}$ Division of KI-ADRC, Department of Neurobiology, Care Sciences and Society, Karolinska Institutet, Stockholm, \\ Sweden \\ ${ }^{\mathrm{g}}$ Neurology Department, Hospital Clínico San Carlos, Madrid, Spain
}

Handling Associate Editor: Alberto Villarejo

Accepted 2 February 2014

\begin{abstract}
. mortality risk included few different dementia diagnoses. diagnosis, adjusting for potential confounding factors. disease and vascular dementia (HR 1.32; 95\% CI 1.22-1.44).

\footnotetext{
${ }^{1}$ Authors responsible for statistical analysis.

*Correspondence to: Sara Garcia-Ptacek, Division of Clinical Geriatrics, Department of Neurobiology, Care Sciences and Society, Karolinska Institutet, Section for Clinical Geriatrics, Novum plan 5, 14186 Stockholm, Sweden. Fax: +46 858585470; E-mail: saragptacek@gmail.com.
}

Background: Knowledge on survival in dementia is crucial for patients and public health planning. Most studies comparing

Objectives: To compare mortality risk in the most frequent dementia disorders in a large cohort of patients with an incident

Methods: 15,209 patients with dementia from the national quality database, Swedish Dementia Registry (SveDem), diagnosed in memory clinics from 2008 to 2011, were included in this study. The impact of age, gender, dementia diagnosis, baseline Mini-Mental State Examination (MMSE), institutionalization, coresidency, and medication on survival after diagnosis were examined using adjusted hazard ratios (HR) with $95 \%$ confidence intervals (CI).

Results: During a mean follow-up of 2.5 years, 4,287 deaths occurred, with 114 (95\% CI 111-117) deaths/1,000 person-years. Adjusted HR of death for men was 1.56 (95\% CI 1.46-1.66) compared to women. Low MMSE, institutionalization, and higher number of medications were associated with higher HR of death. All dementia diagnoses demonstrated higher HR compared to Alzheimer's disease, with vascular dementia presenting the highest crude HR. After adjusting, frontotemporal dementia had the highest risk with a HR of 1.91 (95\% CI 1.52-2.39), followed by Lewy body dementia (HR 1.64; 95\% CI 1.39-1.95), vascular dementia (HR 1.55; 95\% CI 1.42-1.69), Parkinson's disease dementia (HR 1.47; 95\% CI 1.17-1.84), and mixed Alzheimer's 
Conclusion: Worse cognition, male gender, higher number of medications, institutionalization, and age were associated with increased death risk after dementia diagnosis. Adjusted risk was lowest in Alzheimer's disease patients and highest in frontotemporal dementia subjects.

Keywords: Alzheimer's disease, cohort studies, dementia, frontotemporal dementia, mortality, Parkinson's disease, vascular dementia

\section{INTRODUCTION}

Knowledge of mortality risk in dementia is crucial for resource planning and valuable information for patients and families [1,2]. Dementia shortens life expectancy but survival estimates range from 1 to 13 years depending on dementia type, gender, cognitive level, neuropathology, cohort, and study design [2-9]. The availability of data on survival varies greatly by dementia type. Numerous studies have examined mortality in Alzheimer's disease (AD) [1, 6, 7, 9-11] or vascular dementia $(\mathrm{VaD})[11,12]$, although prospective cohorts of incident cases are rarer [1, 7, 9, 10, 13]. Less information exists on other dementia types [2, $14,15]$. Furthermore, comparisons of mortality risk between different dementia diagnoses are infrequent with most studies only including two [7, 14-17] or three diagnoses $[13,18,19]$. Two clinic-based [6, 20] and one population-based study [21] included more dementia types, but large prospective studies of incident cases with a wide range of diagnoses are lacking. AD seems to present longer survival than other dementia types $[2,7,19]$, although others do not confirm this finding $[3,22]$.

A number of factors have been identified as being associated with mortality in dementia. Higher age [1, $3,7,10,22]$ and lower cognition $[4,11,16]$ increase mortality risk, while female gender appears to be protective $[1,3,6,7,12,16,18,22-24]$, although some studies contradict these findings [10, 25] and others propose that the association between gender and mortality might differ by dementia diagnosis $[2,13,26$, 27]. AD patients living alone may suffer higher mortality [28], and comorbidity plays a role and appears reflected in the higher mortality rates associated with institutionalization [5, 7, 22]. For this reason, studies exploring mortality after dementia diagnosis need to take these aspects into consideration.

The Swedish Dementia Registry (SveDem) is a web-based quality initiative that records subjects with incident dementia diagnosis nationwide. To date, the database contains more than 28,000 individual patient entries. The aim of this study is to explore mortality risk in the most frequent dementia disorders in a large prospective cohort. We report mortality risk associated with baseline factors including dementia diagnosis, gender, age, medications, living arrangements, and Mini-Mental State Examination (MMSE) for 15,209 incident dementia patients.

\section{MATERIALS AND PATIENTS}

\section{Study population}

SveDem was created in 2007 to improve quality of care [29-32] for dementia patients throughout Sweden. Newly diagnosed patients meeting ICD-10 [33] criteria for dementia, diagnosed at either memory clinics or primary care, are included in the database. The national guidelines published by the Swedish National Board of Health and Welfare [34] that an established basal dementia work-up be administered to patients with suspicion of dementia are followed in over $85 \%$ of diagnoses [35], and testing can be expanded if necessary. These same guidelines encourage early diagnosis [35]. Quality control of the database is performed by random cross-checks of histories and entries [35]. Based on estimates of dementia incidence in the general Swedish population, in 2011 SveDem captured around $25 \%$ of all new dementia cases in Sweden [35]. The coverage for specialist memory clinics was 93\% [35]. Changing diagnoses within the first year of follow-up are about $5 \%$. SveDem is collated with the national population registry to record deaths.

Dementia diagnoses are coded as $\mathrm{AD}, \mathrm{VaD}, \mathrm{AD}$ and VaD (mixed), Lewy Body Disease (LBD, McKeith criteria [36]), Parkinson's-Disease Dementia (PDD, Movement Disorder Society Task Force criteria [37]), frontotemporal dementia (FTD, Manchester criteria [38]), unspecified dementia (where specific dementia diagnosis is not ascertained), and other dementia types (grouping miscellaneous dementia disorders such as corticobasal degeneration or alcohol related dementias). Simultaneously, age, gender, and baseline MMSE are entered, as are coresidency status (living alone versus cohabiting) and place of residence (home versus institution). The number of medication that the patient takes regularly at the beginning of diagnostic workup comprises all medications that the patient takes that appear in the official Swedish Drug Index [39]. 
This variable is used as a proxy for comorbidity [32, 40], since it has been shown to be better than other medication-based comorbidity scores at predicting morbimortality [40]. The presence of cardiovascular medication, antidepressants, antianxiety, neuroleptics, and sleeping aids is recorded. Cholinesterase inhibitors and N-Methyl-D-aspartate (NMDA) antagonists prescribed upon diagnosis are entered.

For this study, we selected patients included in SveDem who had received a dementia diagnosis from specialist memory clinics from 2008 through 2011 and for whom complete data on dementia diagnosis, age, gender, and survival was available. The decision to exclude primary care was due to later and more irregular inclusion of these units in the registry, uncertainty over national coverage levels and differences in diagnostic process compared to specialist care [30]. Of 15,224 patient entries, $15(0.1 \%)$ patients were excluded for incomplete data on diagnosis, age, gender, or time of death. A total of 15,209 patients were thus included in the analysis. Survival was examined according to the above variables.

To eliminate potential bias from the exclusion of patients from primary care, the final analyses were repeated post-hoc in all 28,722 patients (from both specialist and primary care) included in the register between 2007 and 2012.

\section{Statistical analyses}

Prospective analyses to identify factors associated with mortality risk were performed using Cox proportional hazards regression models and are shown as hazard ratios (HR) of death with $95 \%$ confidence intervals (CI). Kaplan-Meier survival curves and models with time-dependent covariates were employed to test if the assumption of proportional hazards was met. In the instances in which mortality hazards were not proportional, the hazard at the beginning of the observation period and the hazard at day 1,000 of follow-up were calculated in addition to the average hazard over the whole observation period. Personyears (PY) at risk were calculated individually for all patients from dementia diagnosis to date of death or end of follow-up, on February 2013. Standardized mortality ratios (SMR) of the SveDem cohort relative to the general Swedish population were calculated. In order to minimize the number of persons with dementia present in the general Swedish population figures, mortality rates for ICD-10 codes F00 (dementia due to AD), F01 (VaD), F02 (dementia in other disease), F03 (unspecified dementia), and F05 (delirium, not substance induced) were subtracted from the general Swedish population mortality rates [33]. For descriptive statistics, means and standard deviations (SD) are provided where appropriate. SPSS $®$ version 21 and PROC PHREG in the Statistical Analysis System (SAS®) version 9.3 were used for analyses.

Age was explored as a continuous variable and also divided into four categories (under 65, 65-74, 75-84, and 85 and above). The first cut point for age (under 65) was selected to represent the distinction between early and late-onset dementia [41], with further cut points every 10 years of age. Number of medication was categorized as $0-1,2-5,6-9$, and 10 or more habitual drugs at time of diagnostic workup. MMSE score was classified as not assessable, severe (0-9), moderate (10-19), mild (20-24), and slight impairment (25 and over). These categories result from the tertile distribution of MMSE in our sample $(<19,20-24$, and over 25$)$ with a further subdivision in the lowest MMSE category so as not to group patients with very different cognitive performance. Gender, coresidency and place of residence were treated as dichotomous variables.

Crude and age and gender adjusted HR were calculated (results not shown). All presented HR estimates were adjusted for age, gender, dementia diagnosis, MMSE, and number of medication unless otherwise stated. The interaction between gender and dementia type and gender and MMSE were analyzed. A previous study conducted on SveDem showed reduced mortality with cholinesterase inhibitors [42], so additional models were repeated controlling for these medications, as well as for presence or absence (as dichotomous variables) of cardiovascular medication, antidepressants, neuroleptics, antianxiety medication, and sleeping aids.

\section{Standard protocol approvals and patient consent}

The data collection and analysis procedures were approved by the regional ethics committee in Stockholm (approval number 2009/209-31). Patients and caretakers were informed orally and in writing about SveDem, and could decline participation and withdraw consent at any time. Data was collected locally and entered into the web-based database, and coded and anonymized before statistical analysis.

\section{RESULTS}

A total of 15,209 patients were included. Characteristics of the study subjects are shown in Tables 1 and 2 . Fifty-nine percent of the study subjects were women. 
Table 1

Characteristics of study subjects

\begin{tabular}{|c|c|c|c|c|}
\hline & Number of patients (\%) & Deaths & PY at risk & Deaths/ 1000 PY \\
\hline All & $15209(100)$ & 4287 & 37619 & 114 \\
\hline \multicolumn{5}{|l|}{ Gender } \\
\hline Women & $8910(58.6)$ & 2279 & 22602 & 101 \\
\hline Men & $6299(41.4)$ & 2008 & 15017 & 134 \\
\hline \multicolumn{5}{|l|}{ Age } \\
\hline$<65$ & $1018(6.7)$ & 91 & 2857 & 32 \\
\hline $65-74$ & $3434(22.6)$ & 557 & 9089 & 61 \\
\hline $75-84$ & $7307(48.0)$ & 2035 & 18301 & 111 \\
\hline$\geq 85$ & $3450(22.7)$ & 1604 & 7372 & 218 \\
\hline Mean (SD) & $78.1(8.2)$ & & & \\
\hline \multicolumn{5}{|l|}{ MMSE } \\
\hline$\geq 25$ & $4382(28.8)$ & 738 & 11685 & 63 \\
\hline$\overline{20}-24$ & $5515(36.3)$ & 1423 & 13858 & 103 \\
\hline $10-19$ & $4164(27.4)$ & 1559 & 9707 & 161 \\
\hline$<10$ & $395(2.6)$ & 174 & 842 & 207 \\
\hline Not assessable & $384(2.5)$ & 223 & 727 & 307 \\
\hline Missing & $369(2.4)$ & 170 & 800 & 213 \\
\hline Mean (SD) & $21.3(5.1)$ & & & \\
\hline \multicolumn{5}{|l|}{ Coresident } \\
\hline No & $6868(45.2)$ & 2086 & 17024 & 123 \\
\hline Yes & $7791(51.2)$ & 1961 & 19626 & 100 \\
\hline Missing & $550(3.6)$ & 240 & 969 & 248 \\
\hline \multicolumn{5}{|l|}{ Residency } \\
\hline Home & $13870(91.2)$ & 3637 & 34969 & 104 \\
\hline Institution & $1321(8.7)$ & 676 & 2606 & 259 \\
\hline Missing & $18(0.1)$ & 13 & 44 & 295 \\
\hline \multicolumn{5}{|c|}{ Number of medication before diagnosis } \\
\hline$<2$ & $2541(16.7)$ & 434 & 6799 & 64 \\
\hline $2-5$ & $6778(44.6)$ & 1736 & 17260 & 101 \\
\hline $6-9$ & $4148(27.3)$ & 1361 & 9817 & 139 \\
\hline$>10$ & $1306(8.6)$ & 592 & 2770 & 214 \\
\hline Missing & $436(2.9)$ & 164 & 974 & 168 \\
\hline Mean (SD) & $4.7(3.3)$ & & & \\
\hline
\end{tabular}

PY, person-years; SD, standard deviation.

Table 2

Distribution of dementia types, age, and number of medication at diagnosis

\begin{tabular}{lcccrrr}
\hline & Patients n (\%) & Mean age (SD) & Mean medication (SD) & Deaths n & PY at risk & Deaths/1000 PY \\
\hline All & $15,209(100)$ & $78(8.2)$ & $47(3.3)$ & 4,287 & 37,619 & 114 \\
AD & $5,641(37.1)$ & $77(8.3)$ & $3.7(2.9)$ & 1,107 & 15,003 \\
Mixed & $3,732(24.5)$ & $81(6.6)$ & $5.2(3.1)$ & 1,182 & 8,932 \\
VaD & $2,832(18.6)$ & $79(7.6)$ & $6.2(3.23)$ & 1,050 & 6,476 \\
LBD & $461(3.0)$ & $77(7.0)$ & $4.7(2.9)$ & $6.4(3.2)$ & 155 & 1,103 \\
PDD & $283(1.9)$ & $75(6.8)$ & $3.3(2.9)$ & 84 & 658 \\
FTD & $348(2.3)$ & $69(9.3)$ & $5.0(3.5)$ & 81 & 862 \\
Unspecified & $1,559(10.3)$ & $79(8.9)$ & $4.6(3.3)$ & 530 & 3,675 \\
Other & $353(2.3)$ & $73(10.1)$ & 98 & 132 & 141 \\
\hline
\end{tabular}

Distribution of dementia types, age and number of medication at diagnosis. PY, person-years; SD, standard deviation; AD, Alzheimer's disease; Mixed, mixed Alzheimer's disease and vascular dementia; VaD, vascular dementia; LBD, Lewy body dementia; PDD, Parkinson's disease dementia; Unspecified, diagnosis not specified or unknown; Other, any other established diagnosis other than AD, mixed, VaD, LBD, or PDD.

Mean age was 78.1 years (SD 8.2), mean MMSE was 21.3 (SD 5.1), and mean number of medications taken by the patient at the beginning of the diagnostic workup was 4.7 (SD 3.3). Thirty-seven percent were diagnosed as $\mathrm{AD}$ and forty-five percent lived alone.

Patients were followed-up for an average of 2.5 years (range 0 to 1869 days) for a total of $37,619 \mathrm{PY}$ at risk and 4,287 observed deaths (114 deaths/1,000 PY; 95\% CI 111 to 117). The mortality rate was 134 deaths per 1,000 PY among men and 101 among women. Table 3 shows an age and gender adjusted mortality comparison between the cohort and the general Swedish population. Included in Table 4 are HRs of death associated with different variables. Non-proportional 
Table 3

Standardized mortality ratios in SveDem relative to the general Swedish population

\begin{tabular}{|c|c|c|c|c|c|c|c|c|c|}
\hline & \multicolumn{3}{|c|}{ Men } & \multicolumn{3}{|c|}{ Women } & \multicolumn{3}{|c|}{ All } \\
\hline & $\begin{array}{c}\text { Observed deaths } \\
\text { per year }\end{array}$ & SMR & $95 \% \mathrm{CI}$ & $\begin{array}{c}\text { Observed deaths } \\
\text { per year }\end{array}$ & SMR & $95 \% \mathrm{CI}$ & $\begin{array}{c}\text { Observed deaths } \\
\text { per year }\end{array}$ & SMR & $95 \%$ CI \\
\hline $45-54$ & 2 & 14.29 & $1.71-51.57$ & 1 & 11.11 & $0.28-61.89$ & 3 & 12.46 & $2.58-36.43$ \\
\hline $55-64$ & 6 & 2.04 & $0.75-4.43$ & 9 & 4.33 & $1.98-8.23$ & 15 & 2.96 & $1.66-5.47$ \\
\hline $65-74$ & 86 & 3.07 & $2.46-3.79$ & 53 & 2.61 & $1.95-3.41$ & 139 & 2.84 & $2.39-3.35$ \\
\hline $75-84$ & 264 & 1.61 & $1.42-1.81$ & 264 & 1.75 & $1.54-1.97$ & 528 & 1.66 & $1.53-1.81$ \\
\hline$>85$ & 251 & 1.66 & $1.46-1.88$ & 340 & 1.80 & $1.61-2.00$ & 591 & 1.21 & $1.11-1.31$ \\
\hline All ages & 609 & 1.76 & $1.62-1.90$ & 667 & 1.84 & $1.70-1.98$ & 1276 & 1.49 & $1.41-1.58$ \\
\hline
\end{tabular}

Comparison of ratio of observed to expected deaths in SveDem relative to the general Swedish population. Population data was obtained from Socialstyrelsen (Swedish National Board of Health and Welfare) for 2011. Observed deaths per year in each gender and age group in SveDem appear in the first column. Standardized mortality ratios (SMR) and 95\% confidence intervals (CI) are shown.

Table 4

Results of Cox proportional hazard regression examining mortality associated to baseline factors

\begin{tabular}{|c|c|c|}
\hline & $\mathrm{HR}(95 \% \mathrm{CI})$ & $p$-value \\
\hline \multicolumn{3}{|l|}{ Gender } \\
\hline Women & ref & \\
\hline Men & $1.56(1.46-1.66)$ & $<0.000$ \\
\hline \multicolumn{3}{|l|}{ Age } \\
\hline$<65$ & ref & \\
\hline $65-74$ & $1.96(1.57-2.44)$ & $<0.000$ \\
\hline $75-84$ & $3.32(2.72-4.17)$ & $<0.000$ \\
\hline$\geq 85$ & $6.17(4.97-7.65)$ & $<0.000$ \\
\hline \multicolumn{3}{|l|}{ MMSE } \\
\hline$\geq 25$ & ref & \\
\hline $20-24$ & $1.45(1.32-1.58)$ & $<0.000$ \\
\hline $10-19$ & $2.14(1.96-2.34)$ & $<0.000$ \\
\hline $0-9$ & $2.91(2.47-3.44)$ & $<0.000$ \\
\hline Not assessable & $3.72(3.19-4.35)$ & $<0.000$ \\
\hline \multicolumn{3}{|l|}{ Coresident } \\
\hline No & ref & \\
\hline Yes & $1.02(0.96-1.09)$ & 0.485 \\
\hline \multicolumn{3}{|l|}{ Residency } \\
\hline Home & ref & \\
\hline Institution & $1.42(1.27-1.57)$ & $<0.000$ \\
\hline \multicolumn{3}{|c|}{ Number of medications } \\
\hline $0-1$ & ref & \\
\hline $2-5$ & $1.25(1.12-1.39)$ & $<0.000$ \\
\hline $6-9$ & $1.53(1.37-1.71)$ & $<0.000$ \\
\hline$\geq 10$ & $2.14(1.88-2.44)$ & $<0.000$ \\
\hline
\end{tabular}

Results of Cox proportional hazard regression examining mortality associated to baseline factors. Results are given as hazard ratios (HR), 95\% confidence intervals (CI) for relative mortality, and $p$-values for parameter estimates (Cox regression) adjusted for all baseline factors as they appear on the table, as well as by dementia diagnosis. First category of each variable serves as reference (ref).

hazard ratios were detected for LBD, unspecified and other dementia types, so time-dependent covariates for these diagnoses were included in the model (Table 5).

\section{Mortality by gender, age, and baseline characteristics}

Men had an increased risk of death compared to women in crude and adjusted analyses (Table 4). Similar gender differences were obtained when data was stratified by MMSE. The interaction terms between gender and MMSE, and gender and dementia type were non-significant. When analyses were stratified by diagnosis, men had significantly higher mortality risk than women in all diagnostic groups, except in FTD where there was no association (HR 1.02, 95\% CI 0.64-1.61, $p=0.94)$ and in LBD where differences were not statistically significant (HR $1.41,95 \%$ CI $0.96-2.07, p=0.077)$. The largest difference between men and women occurred in PDD with a HR for men of 
Table 5

Hazard ratios (HR) of death associated with dementia diagnosis compared to Alzheimer's disease (AD)

\begin{tabular}{|c|c|c|c|c|c|}
\hline & & \multicolumn{2}{|c|}{ Crude } & \multicolumn{2}{|c|}{ Adjusted } \\
\hline & & $\mathrm{HR}(95 \% \mathrm{CI})$ & $p$-value & $\mathrm{HR}(95 \% \mathrm{CI})$ & $p$-value \\
\hline $\mathrm{AD}$ & & ref & & ref & \\
\hline Mixed & & $1.84(1.70-2.00)$ & $<0.001$ & $1.33(1.22-1.44)$ & $<0.001$ \\
\hline $\mathrm{VaD}$ & & $2.27(2.09-2.47)$ & $<0.001$ & $1.56(1.43-1.70)$ & $<0.001$ \\
\hline PDD & & $1.80(1.44-2.24)$ & $<0.001$ & $1.47(1.17-1.84)$ & $<0.001$ \\
\hline FTD & & $1.29(1.03-1.62)$ & 0.026 & $1.91(1.52-2.40)$ & $<0.001$ \\
\hline \multirow[t]{3}{*}{ LBD } & Average & $1.96(1.66-2.32)$ & $<0.001$ & $1.75(1.47-2.08)$ & $<0.001$ \\
\hline & Beginning of follow-up & $1.49(1.09-2.05)$ & 0.013 & $1.23(0.89-1.68)$ & 0.211 \\
\hline & After 1000 days & $2.22(1.83-2.71)$ & $<0.001$ & $1.89(1.55-2.31)$ & $<0.001$ \\
\hline \multirow[t]{3}{*}{ Unspecified } & Average & $1.99(1.79-2.20)$ & $<0.001$ & $1.43(1.29-1.59)$ & $<0.001$ \\
\hline & Beginning of follow-up & $2.52(2.13-2.99)$ & $<0.001$ & $1.75(1.47-2.08)$ & $<0.001$ \\
\hline & After 1000 days & $1.74(1.52-1.98)$ & $<0.001$ & $1.28(1.12-1.46)$ & $<0.001$ \\
\hline \multirow[t]{3}{*}{ Other } & Average & $1.45(1.18-1.79)$ & $<0.001$ & 1.35 (1.09-1.67) & $<0.001$ \\
\hline & Beginning of follow-up & $2.05(1.43-2.94)$ & $<0.001$ & $1.92(1.33-2.77)$ & $<0.001$ \\
\hline & After 1000 days & $1.22(0.92-1.61)$ & 0.169 & $1.13(0.85-1.49)$ & 0.406 \\
\hline
\end{tabular}

HR, 95\% confidence intervals (CI), and $p$-values comparing different dementia diagnosis with AD. Results were obtained from Cox regression analysis and Cox regression with time-dependent covariates. Crude results in left-hand columns. In right-hand columns, HRs adjusted for age, gender, Mini-Mental State Exam, coresident, residential setting, and number of medication at the beginning of diagnostic workup. Lewy body dementia (LBD), unspecified dementia, and other dementia diagnoses had significantly non-proportional hazards compared to AD: for these, average HR for the whole follow-up period is given together with the HR at the beginning of follow-up and HR at 1,000 days of follow-up. Mixed, mixed Alzheimer's disease and vascular dementia; VaD, vascular dementia; PDD, Parkinson's disease dementia; Unspecified, diagnosis not specified or unknown; Other, any other established diagnosis other than $\mathrm{AD}$, mixed, VaD, LBD, or PDD; ref, AD group served as a reference category.

$1.71(95 \%$ CI 1.04-2.79) and in other dementias (HR $1.73 ; 95 \%$ CI 1.12-2.66) (data not presented in tables).

In crude analyses, each additional year of age increased mortality by $8 \%$. In the final adjusted analysis, compared to patients under 65, each subsequent category was associated with increased mortality risk (Table 4).

Compared with the patients scoring high on MMSE $(\geq 25)$, those scoring lower had higher risk of death, with risk increasing with decreasing cognition (Table 4). Patients in whom MMSE could not be administered, judged not assessable, had the highest risk (HR 3.72, 95\% CI 3.19-4.35).

Higher number of medications was associated with increased mortality, with the highest HR occurring among patients taking 10 or more medications (Table 4). Living in an institution was associated with increased risk of death (Table 4).

\section{Mortality by dementia diagnosis}

As shown in the survival functions in Fig. 1, lower mortality risk was observed among AD patients compared to all other dementia diagnoses. In Cox regression, the highest crude $\mathrm{HR}$ corresponded to $\mathrm{VaD}$ (HR 2.27, 95\% CI 2.08-2.47) (Table 5). This relationship remained unchanged after adjusting for gender. After adjusting for age and gender, PDD had the highest risk (Fig. 1A). However, after number of medication was introduced as a covariate, FTD had the highest risk.

When the whole follow-up period was examined, in the final multivariate model, all other dementia diagnoses were significantly associated with higher mortality risk compared to AD (Table 5; Fig. 1B). Mixed dementia presented a HR of 1.32 (95\% CI 1.22-1.44), intermediate between $\mathrm{AD}$ and $\mathrm{VaD}$, while FTD presented the highest risk (HR 1.91; 95\% CI 1.52-2.39). LBD, unspecified dementia, and other dementia had significant non-proportional mortality hazards, meaning that death risk diverged from $\mathrm{AD}$ over time. For LBD, the average death risk over the whole observation period was significantly higher than for AD. Death risk relative to $\mathrm{AD}$ increased significantly over time ( $p$-value for change over time is not presented). A statistically significant difference was not demonstrated at the beginning of the observation period, but a higher HR for death was evident at 1,000 days.

For unspecified and other dementia diagnoses, mortality risk relative to AD decreased over time (Table 5).

Additional adjustment for cholinesterase inhibitors and individual medications (such as cardiovascular treatment or antidepressants) as potential confounding factors showed no substantial changes in results (results not presented).

Post-hoc analyses were rerun on all 28,704 patients in the register (including primary care). The gradation of mortality risk relative to $\mathrm{AD}$ did not change, with 

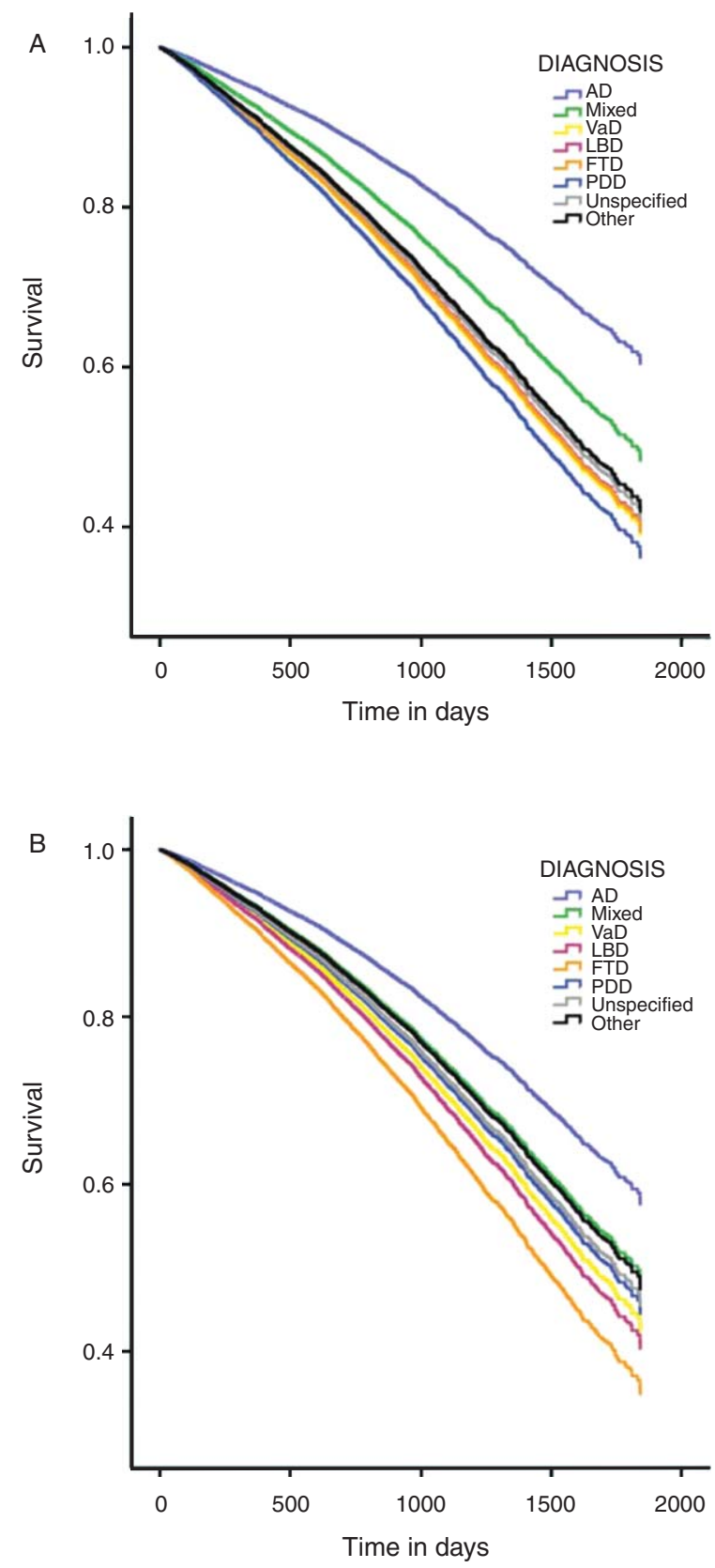

Fig. 1. Age and gender adjusted survival function from Cox hazard regression. Y-axis: estimated survival percentages. Xaxis: number of days. When not adjusting for age, patients with vascular dementia ( $\mathrm{VaD})$ has the highest mortality risk. A) Age-and-gender adjusted mortality risk was highest for Parkinson's disease with dementia (PDD). B) In the final model, after adjusting for age, gender, Mini-Mental State Exam, coresident, residential setting and number of medication at the beginning of diagnostic workup, frontotemporal dementia (FTD) presented the highest risk. AD, Alzheimer's disease; Mixed, mixed Alzheimer's disease and vascular dementia; LBD, Lewy body dementia; Unspecified, unspecified dementia; Other, other dementia diagnoses.
HRs remaining roughly similar to those obtained from the cohort containing only specialist memory clinic patients.

\section{DISCUSSION}

The results of this study show that worse cognition, male gender, institutionalization, higher number of medications, and higher age are associated with increased mortality after dementia diagnosis regardless of dementia type. AD exhibits better survival than other dementias while FTD has the highest mortality after adjusting for age, gender, MMSE, number of medication, institutionalization, and coresidency.

Our incident dementia cohort presented a mortality rate of 134 deaths per 1000 PY among men and 101 among women. The age and gender adjusted SMR indicated excess mortality in SveDem compared to the general Swedish population (Table 3). Caution must be taken in interpreting these results as higher age groups in the general Swedish population are likely to contain a significant proportion of persons with dementia. We have attempted to control for this by subtracting the mortality rates corresponding to the ICD-10 codes for dementia and non-alcoholic delirium (F00, F01, F02, F03, and F05) from the general rates, but this only accounts for a fraction of these persons. Our finding of 111 deaths per $1000 \mathrm{PY}$ in the 75-84 age group compares to the 172 deaths per 1000 PY reported in patients with incident stroke in the same age group in Sweden [43]. Our rates were lower than those described in other dementia cohorts. In the same age group, the NEDICES cohort [11], reported mortality rates of 224 and 166 deaths per 1000 PY for men and women respectively, which is higher than the 138 deaths per 1000 PY for men and 94 for women observed in our study. This is despite the NEDICES cohort being a prevalent cohort in which length bias might have suggested lower mortality rates. A Swedish study conducted on the +77 group within the Kungsholmen [7] project on incident dementia reported mortality rates for the 77-84 age group of 288 deaths per 1000 PY for men and 175 for women. Both these studies are population-based and comparisons with our register-based study must be tentative. Both had longer follow-up than ours, which might lower our mortality rates since a proportion of patients would foreseeably die in later years. Additionally, both these studies were conducted in the 1990s: greater quality of care and changes in clinical practice with diagnostic boundaries shifting toward the mild end of the dementia spectrum might reduce mortality in present-day patients. 
Lower mortality risk was found in women across all cognitive levels. Our findings confirm the general trend in published literature of lower mortality with female gender $[1,3,6,7,12,16,18,22-24]$. This trend is not without exceptions $[10,25]$, and some authors have found a different gender effect with different dementia diagnoses $[2,13,26,27]$ with women afflicted with $\mathrm{VaD}$ [13] or LBD [44] faring worse. In our study, lower risk for women was apparent in all dementia types, except FTD and LBD, where results were not statistically significant. Insufficient sample size or different gender effects in these dementia types could explain these results.

Not surprisingly, age is associated with increased mortality in dementia [1, 3, 7, 10, 22]. The study on the +77 subgroup of the Swedish Kungsholmen project [7] reported a $10 \%$ increase in mortality risk for each 1 -year increase in age. This compares with our finding of $8 \%$ per year.

The present study indicates that worse baseline cognition, as represented by MMSE, is associated with higher mortality risk. This increased risk is already apparent from the rather mild levels of cognitive impairment represented by the MMSE 20-24 group and increases with further decline. Previously, some studies only demonstrated increased mortality at severe stages of cognitive decline $[11,16]$ while a metaanalysis by Dewey et al. [4] found increased mortality even with moderate impairment. Despite not having a non-demented control group for comparison, our finding of 63 deaths per $1000 \mathrm{PY}$ in the MMSE $\geq 25$ and 103 deaths per 1000 PY in the 20-24 category is not negligible, and suggests that even mild dementia is associated with increased mortality. The group deemed "non assessable" presented the highest risk. If most of these patients are incapable of taking the test because of low cognition, this would hint at a floor effect in the MMSE test and would signify that mortality continues to increase when cognitive deterioration progresses below the test threshold. However, this group is heterogeneous, comprising patients who refuse to take the test and those who cannot due to advanced dementia or coexisting conditions, which might be associated with increased mortality. Our existing data are insufficient to support one hypothesis over the other.

Comorbidity and disability have been reported to increase mortality after dementia diagnosis [5, 7, 22]. The higher risk among institutionalized patients reflects this. In our cohort, the number of medications was associated with increased mortality. We recently showed that treatment with cholinesterase-inhibitors for $\mathrm{AD}$ was associated with reduced mortality in $\mathrm{AD}$ patients in SveDem [42], so this variable was included in the analysis as a potential confounder. The presence of cholinesterase inhibitors in the models did not alter the other results, and neither did other individual medication types.

In our large national cohort, different dementia diagnoses were associated with significantly different mortality rates, with all dementia types showing increased risk relative to $\mathrm{AD}$. VaD displayed the highest crude mortality risk, but FTD was the most lethal dementia after adjusting. This could reflect the fact that FTD disproportionally affects younger individuals who suffer from less comorbidity. In our cohort, the average age in the FTD group was lower than in $\mathrm{AD}$, and they took less medication (Table 2). The FTD group had few patients in the over 85 group $(n=7)$, so we repeated Cox regression analyses only with patients under 85 , with similar results.

The lower mortality associated with AD remained after stratifying for gender and adjusting for potential covariates, including MMSE, age, and medications. As expected given the etiopathology of the condition, mixed dementia presented a HR that was significantly higher than $\mathrm{AD}$ but lower than $\mathrm{VaD}$. The difference between $\mathrm{AD}$ and LBD increased over time. The heterogeneity of the "other dementia" category makes it harder to interpret results. Previous literature reports conflicting results on the influence of different dementia diagnosis on mortality, although most publications report either lower risk with $\mathrm{AD}[2,6,7]$ or fail to demonstrate a significant difference $[11,18,19,21$, 22]. Most studies compare $\mathrm{AD}$ and $\mathrm{VaD}$ (sometimes including mixed dementia) $[7,11,18,45,46]$ or $\mathrm{AD}$ with $\operatorname{LBD}[9,15,25,26,47]$ but few analyze a wider spectrum of diagnoses $[6,9,20]$. When comparing $\mathrm{AD}$ and $\mathrm{VaD}$, most studies either fail to demonstrate a difference in survival $[7,11,18,45,46]$ or suggest lower risk with $\mathrm{AD}[12,27]$. The cardiovascular risk factors that contribute to a $\mathrm{VaD}$ diagnosis would naturally drive up cardiovascular mortality, the main cause of death across cohorts according to previous publications [11]. Landi and colleagues [48] found that $\mathrm{AD}$ patients had fewer comorbid conditions than $\mathrm{VaD}$, even after excluding cardiovascular risk factors, making $\mathrm{AD}$ a "healthier" demented cohort. The only proxy for co-morbidity available in our study is the number of medication at the beginning of workup, which was 3.7 in the AD group compared to the sample average of 4.7 and the 6.2 average among $\mathrm{VaD}$. This might support the idea of less co-morbidity in the AD group. However, our survival analyses were controlled for number of medication. The same analyses run without 
controlling for number of medication showed similar results, with a slight increase in the $\mathrm{HR}$ for $\mathrm{VaD}$ and PDD. Cholinesterase inhibitors were also examined and did not alter results. In a previous study, $\mathrm{VaD}$ was found to increase mortality relative to $\mathrm{AD}$, with mixed dementia presenting intermediate risk [13]. These findings mirror our own and fit with the presumed etiopathology of mixed dementia as a condition with both $\mathrm{AD}$ and $\mathrm{VaD}$ features.

Previous studies found that LBD presented worse prognosis than $\operatorname{AD}[15,26]$, even after controlling for cognitive level [25], although others find no differences in survival or age at death [47]. In our cohort, multivariate Cox analyses run with LBD as index category showed a significantly increased risk relative to AD and mixed dementia, but no difference with $\mathrm{VaD}$ or PDD diagnoses. This could reflect a lack of statistical power, or physiopathological similarities between the LBD and PDD diagnoses.

Frontotemporal degeneration survival estimates range from 3 [49] to 9.5 [50] years but vary depending on variant type [49], presence of motor neuron disease [49], and symptoms at onset [50]. FTD survival compares unfavorably to AD in most studies $[6,20]$. With our available data, we cannot ascertain the variant type proportion in our FTD group.

No statistically significant differences were found between mortality risk in different dementia types in a population-based cohort of 828 patients that examined $\mathrm{AD}, \mathrm{VaD}, \mathrm{LBD}$, and combined dementias [21]. This was probably due to the low number of dementia cases $(n=275)$, particularly LBD $(n=12)$. Two clinic-based studies have compared a wider variety of diagnoses. Koedam et al. [20] found lower mortality risk for AD, with the highest HR for LBD, although differences in risk estimates were not significant. Steenland and colleagues [6] found the highest mortality in motor neuron disease, followed by FTD, LBD, Parkinson's disease (PD), AD, and mild cognitive impairment compared to controls. Their PD group included PD patients without cognitive impairment, but otherwise their gradation of mortality by disease type matches our findings.

Weaknesses of this study are the absence of cognitively normal controls and its descriptive nature. Our follow-up time, from January 2008 to February 2013, might have been too short. SveDem contains a lower proportion of $\mathrm{AD}$ diagnosis than previously observed [51], but this is probably due to classifying mixed dementia as separate from AD. Length bias refers to the overestimation of survival due to failure to include patients with rapidly progressive disease who die before recruitment, and appears whenever studies mix incident and prevalent cases [3]. Our inclusion of only incident cases should help control this bias. However, neurodegeneration in dementia is thought to begin years or decades before diagnosis, and determining the exact moment of dementia incidence is probably impossible.

The exclusion of patients from primary care could be a source of bias since the patient population differs from specialist care. Primary care diagnoses extremely few patients with PDD or LBD, for example, so joint analyses of primary and specialist patient groups are problematic. Still, analyses were repeated with all 28,722 patients from both memory clinics and primary care included in SveDem from 2007 and 2012: the gradation of mortality by diagnosis, and even the hazard ratios for different factors remained roughly similar. This should assuage concerns of a biased sample.

The reliability of clinical diagnoses of dementia is always a concern in studies of this nature. To complicate matters, the correlation between clinical diagnosis and pathology is not straightforward [52]. The fact remains that in clinical practice patients are diagnosed and treated with a clinical diagnosis. As such, mortality information from SveDem is relevant to the clinical process.

In 2011, SveDem captured an estimated $25 \%$ of all new dementia cases in Sweden. These calculations are based on the estimated incidence of dementia, but the percentage of new dementia cases who undergo dementia work-up and receive a diagnosis each year is unknown [35]. The coverage in specialist care is 93\%, making SveDem uniquely representative among this population. However, there might be baseline differences between the population in SveDem and populations with incident dementia who do not receive a diagnosis or who are diagnosed by units not included in SveDem.

Strengths of our study are its inclusion of a wider variety of dementia disorders than previously published and that all diagnoses were made in specialist clinics, improving reliability. A review of literature indicates that this is the largest prospective study of its type examining mortality in incident dementia.

\section{CONCLUSION}

In our study, male gender, higher age, institutionalization, lower cognitive status measured by MMSE, and higher number of medications before dementia diagnosis were independently associated with increased mortality risk. VaD showed the highest crude 
mortality rate and FTD the highest adjusted mortality. This might translate the fact that FTD predominantly affects younger and healthier individuals.

Our findings concur with published literature showing that $\mathrm{AD}$ has a more benign course than other dementias, but the reasons remain unclear. Coexistent comorbidities or factors relating to the cognitive profile of different diseases might play a role. The underlying neurodegenerative disease process might directly impact mortality.

\section{ACKNOWLEDGMENTS}

The authors are grateful to SveDem (http://www. svedem.se) for providing data for this study as well as many thanks to all participants in SveDem (patients, caregivers, and staff). This study was supported financially by the Swedish Brain Power network, the Swedish association of local authorities and regions, the Stockholm County Council and Alzheimerfonden. Sara Garcia-Ptacek had full access to all the data in the study and takes responsibility for the integrity of the data and the accuracy of the data analysis. Sara GarciaPtacek, Bahman Farahmand, Dorota Religa, and Maria Eriksdotter contributed to study concept and design and analysis and interpretation of data. Maria Luz Cuadrado and Ingemar Kåreholt participated in analysis and interpretation of data. All authors contributed to drafting the article and revising for intellectual content as well as approved the final version. Conflicts of interest: Sara Garcia Ptacek is supported by SveDem.

Authors' disclosures available online (http://www. j-alz.com/disclosures/view.php?id=2135).

\section{REFERENCES}

[1] Ganguli M, Dodge HH, Shen C, Pandav RS, DeKosky ST (2005) Alzheimer disease and mortality: A 15-year epidemiological study. Arch Neurol 62, 779-784.

[2] Brodaty H, Seeher K, Gibson L (2012) Dementia time to death: A systematic literature review on survival time and years of life lost in people with dementia. Int Psychogeriatr 24, 1034-1045.

[3] Wolfson C, Wolfson DB, Asgharian M, M'Lan CE, Ostbye T, Rockwood K, Hogan DB (2001) A reevaluation of the duration of survival after the onset of dementia. NEngl J Med 344, 1111-1116.

[4] Dewey ME, Saz P (2001) Dementia, cognitive impairment and mortality in persons aged 65 and over living in the community: A systematic review of the literature. Int J Geriatr Psychiatry 16, 751-761.

[5] Koller D, Kaduszkiewicz H, van den Bussche H, Eisele M, Wiese B, Glaeske G, Hoffmann F (2012) Survival in patients with incident dementia compared with a control group: A five-year follow-up. Int Psychogeriatr 24, 1522-1530.
[6] Steenland K, MacNeil J, Seals R, Levey A (2010) Factors affecting survival of patients with neurodegenerative disease. Neuroepidemiology 35, 28-35.

[7] Aguero-Torres H, Fratiglioni L, Guo Z, Viitanen M, Winblad B (1999) Mortality from dementia in advanced age: A 5-year follow-up study of incident dementia cases. J Clin Epidemiol 52, 737-743.

[8] Rait G, Walters K, Bottomley C, Petersen I, Iliffe S, Nazareth I (2010) Survival of people with clinical diagnosis of dementia in primary care: Cohort study. BMJ 341, c3584.

[9] Lonnroos E, Kyyronen P, Bell JS, van der Cammen TJ, Hartikainen S (2013) Risk of death among persons with Alzheimer's disease: A national register-based nested casecontrol study. J Alzheimers Dis 33, 157-164.

[10] Brookmeyer R, Corrada MM, Curriero FC, Kawas C (2002) Survival following a diagnosis of Alzheimer disease. Arch Neurol 59, 1764-1767.

[11] Villarejo A, Benito-Leon J, Trincado R, Posada IJ, PuertasMartin V, Boix R, Medrano MR, Bermejo-Pareja F (2011) Dementia-associated mortality at thirteen years in the NEDICES Cohort Study. J Alzheimers Dis 26, 543-551.

[12] Knopman DS, Rocca WA, Cha RH, Edland SD, Kokmen E (2003) Survival study of vascular dementia in Rochester, Minnesota. Arch Neurol 60, 85-90.

[13] Fitzpatrick AL, Kuller LH, Lopez OL, Kawas CH, Jagust W (2005) Survival following dementia onset: Alzheimer's disease and vascular dementia. J Neurol Sci 229-230, 43-49.

[14] Roberson ED, Hesse JH, Rose KD, Slama H, Johnson JK, Yaffe K, Forman MS, Miller CA, Trojanowski JQ, Kramer JH, Miller BL (2005) Frontotemporal dementia progresses to death faster than Alzheimer disease. Neurology 65, 719-725.

[15] Hanyu H, Sato T, Hirao K, Kanetaka H, Sakurai H, Iwamoto T (2009) Differences in clinical course between dementia with Lewy bodies and Alzheimer's disease. Eur J Neurol 16, 212217.

[16] Aevarsson O, Svanborg A, Skoog I (1998) Seven-year survival rate after age 85 years: Relation to Alzheimer disease and vascular dementia. Arch Neurol 55, 1226-1232.

[17] Jellinger KA, Wenning GK, Seppi K (2007) Predictors of survival in dementia with Lewy bodies and Parkinson dementia. Neurodegener Dis 4, 428-430.

[18] Bruandet A, Richard F, Bombois S, Maurage CA, Deramecourt V, Lebert F, Amouyel P, Pasquier F (2009) Alzheimer disease with cerebrovascular disease and vascular dementia: Clinical features and course compared with Alzheimer disease. J Neurol Neurosurg Psychiatry 80, 133-139.

[19] Barclay LL, Zemcov A, Blass JP, Sansone J (1985) Survival in Alzheimer's disease and vascular dementias. Neurology 35 , 834-840.

[20] Koedam EL, Pijnenburg YA, Deeg DJ, Baak MM, van der Vlies AE, Scheltens P, van der Flier WM (2008) Early-onset dementia is associated with higher mortality. Dement Geriatr Cogn Disord 26, 147-152.

[21] Matsui Y, Tanizaki Y, Arima H, Yonemoto K, Doi Y, Ninomiya T, Sasaki K, Iida M, Iwaki T, Kanba S, Kiyohara Y (2009) Incidence and survival of dementia in a general population of Japanese elderly: The Hisayama study. J Neurol Neurosurg Psychiatry 80, 366-370.

[22] Xie J, Brayne C, Matthews FE (2008) Survival times in people with dementia: Analysis from population based cohort study with 14 year follow-up. BMJ 336, 258-262.

[23] Rountree SD, Chan W, Pavlik VN, Darby EJ, Doody RS (2012) Factors that influence survival in a probable Alzheimer disease cohort. Alzheimers Res Ther 4, 16. 
[24] Jagger C, Clarke M, Stone A (1995) Predictors of survival with Alzheimer's disease: A community-based study. Psychol Med 25, 171-177.

[25] Stubendorff K, Hansson O, Minthon L, Londos E (2011) Differences in survival between patients with dementia with Lewy bodies and patients with Alzheimer's disease-measured from a fixed cognitive level. Dement Geriatr Cogn Disord 32 , 408-416.

[26] Magierski R, Kloszewska I, Sobow TM (2010) The influence of vascular risk factors on the survival rate of patients with dementia with Lewy bodies and Alzheimer disease. Neurol Neurochir Pol 44, 139-147.

[27] Ostbye T, Hill G, Steenhuis R (1999) Mortality in elderly Canadians with and without dementia: A 5-year follow-up. Neurology 53, 521-526.

[28] Nourhashemi F, Amouyal-Barkate K, Gillette-Guyonnet S, Cantet C, Vellas B (2005) Living alone with Alzheimer's disease: Cross-sectional and longitudinal analysis in the REAL.FR Study. J Nutr Health Aging 9, 117-120.

[29] Religa D, Spangberg K, Wimo A, Edlund AK, Winblad B, Eriksdotter-Jonhagen M (2012) Dementia diagnosis differs in men and women and depends on age and dementia severity: Data from SveDem, the Swedish Dementia Quality Registry. Dement Geriatr Cogn Disord 33, 90-95.

[30] Wimo A, Religa D, Spangberg K, Edlund AK, Winblad B, Eriksdotter M (2013) Costs of diagnosing dementia: Results from SveDem, the Swedish Dementia Registry. Int J Geriatr Psychiatry 28, 1039-1044.

[31] Fereshtehnejad SMRD, Westman E, Aarsland D, Lökk J, Eriksdotter M (2013) Comparison of dementia with Lewy bodies with Parkinson's disease dementia in the Swedish Dementia Quality Registry (SveDem). Neuropsychiatr Dis Treat 9, 927-935.

[32] Johnell K, Religa D, Eriksdotter M (2013) Differences in drug therapy between dementia disorders in the Swedish Dementia Registry: A nationwide study of over 7,000 patients. Dement Geriatr Cogn Disord 35, 239-248.

[33] World Health, Organization (1992) The ICD-10 classification of mental and behavioural disorders: Clinical descriptions and diagnostic guidelines, World Health Organization, Geneva.

[34] Socialstyrelsen, Nationella riktlinjer för vård och omsorg vid demenssjukdom 2010-stöd för styrning och ledning (2010) (Swedish National Board of Health and Welfare. National Guidelines for Care in Cases of Dementia), http://www.socialstyrelsen.se, Accessed September 25, 2013.

[35] SveDem styrgruppen, Årsrapport SveDem 2011 (2011) (SveDem stirring committee. Yearly report on SveDem 2011). http://www.ucr.uu.se/svedem/index.php/om-svedem/ arsrapporter, Updated November 28, 2012, Accessed September 25,2013

[36] McKeith IG (2006) Consensus guidelines for the clinical and pathologic diagnosis of dementia with Lewy bodies (DLB): Report of the Consortium on DLB International Workshop. J Alzheimers Dis 9, 417-423.

[37] Emre M, Aarsland D, Brown R, Burn DJ, Duyckaerts C, Mizuno Y, Broe GA, Cummings J, Dickson DW, Gauthier S, Goldman J, Goetz C, Korczyn A, Lees A, Levy R, Litvan I, McKeith I, Olanow W, Poewe W, Quinn N, Sampaio C, Tolosa E, Dubois B (2007) Clinical diagnostic criteria for dementia associated with Parkinson's disease. Mov Disord 22, 1689-1707; quiz 1837.
[38] (1994) Clinical and neuropathological criteria for frontotemporal dementia. The Lund and Manchester Groups. J Neurol Neurosurg Psychiatry 57, 416-418.

[39] FASS, http://www.fass.se/LIF/startpage?5, Accessed November 28, 2013.

[40] Schneeweiss S, Seeger JD, Maclure M, Wang PS, Avorn J, Glynn RJ (2001) Performance of comorbidity scores to control for confounding in epidemiologic studies using claims data. Am J Epidemiol 154, 854-864.

[41] Rossor MN, Fox NC, Mummery CJ, Schott JM, Warren JD (2010) The diagnosis of young-onset dementia. Lancet Neurol 9, 793-806.

[42] Nordstrom P, Religa D, Wimo A, Winblad B, Eriksdotter M (2013) The use of cholinesterase inhibitors and the risk of myocardial infarction and death: A nationwide cohort study in subjects with Alzheimer's disease. Eur Heart J 34, 25852591.

[43] Henriksson KM, Farahmand B, Johansson S, Asberg S, Terent A, Edvardsson N (2010) Survival after stroke-the impact of CHADS2 score and atrial fibrillation. Int J Cardiol 141, 18-23.

[44] Williams MM, Xiong C, Morris JC, Galvin JE (2006) Survival and mortality differences between dementia with Lewy bodies vs Alzheimer disease. Neurology 67, 1935-1941.

[45] Hier DB, Warach JD, Gorelick PB, Thomas J (1989) Predictors of survival in clinically diagnosed Alzheimer's disease and multi-infarct dementia. Arch Neurol 46, 1213-1216.

[46] Rockwood K, Wentzel C, Hachinski V, Hogan DB, MacKnight C, McDowell I (2000) Prevalence and outcomes of vascular cognitive impairment. Vascular Cognitive Impairment Investigators of the Canadian Study of Health and Aging. Neurology 54, 447-451.

[47] Walker Z, Allen RL, Shergill S, Mullan E, Katona CL (2000) Three years survival in patients with a clinical diagnosis of dementia with Lewy bodies. Int J Geriatr Psychiatry 15, 267273.

[48] Landi F, Gambassi G, Lapane KL, Sgadari A, Mor V, Bernabei R (1999) Impact of the type and severity of dementia on hospitalization and survival of the elderly. The SAGE Study Group. Dement Geriatr Cogn Disord 10, 121-129.

[49] Kang SJ, Cha KR, Seo SW, Kim EA, Cheong HK, Kim EJ, Na DL, Jeong JH (2010) Survival in frontotemporal lobar degeneration in a Korean population. Alzheimer Dis Assoc Disord 24, 339-342.

[50] Garcin B, Lillo P, Hornberger M, Piguet O, Dawson K, Nestor PJ, Hodges JR (2009) Determinants of survival in behavioral variant frontotemporal dementia. Neurology 73, 1656-1661.

[51] Fratiglioni L, Launer LJ, Andersen K, Breteler MM, Copeland JR, Dartigues JF, Lobo A, Martinez-Lage J, Soininen H, Hofman A (2000) Incidence of dementia and major subtypes in Europe: A collaborative study of population-based cohorts. Neurologic Diseases in the Elderly Research Group. Neurology 54, S10-S15.

[52] Hyman BT, Phelps CH, Beach TG, Bigio EH, Cairns NJ, Carrillo MC, Dickson DW, Duyckaerts C, Frosch MP, Masliah E, Mirra SS, Nelson PT, Schneider JA, Thal DR, Thies B, Trojanowski JQ, Vinters HV, Montine TJ (2012) National Institute on Aging-Alzheimer's Association guidelines for the neuropathologic assessment of Alzheimer's disease. Alzheimers Dement 8, 1-13. 